\title{
BMJ Open Effects of a workplace prevention programme for problem gambling: study protocol for a cluster randomised controlled trial
}

\author{
Jonas Rafi, Ekaterina Ivanova, Alexander Rozental, Per Carlbring
}

To cite: Rafi J, Ivanova E, Rozental A, et al. Effects of a workplace prevention programme for problem gambling: study protocol for a cluster randomised controlled trial. BMJ Open 2017;7:e015963. doi:10.1136/ bmjopen-2017-015963

- Prepublication history and additional material for this paper are available online. To view please visit the journal online (http://dx.doi.org/10.1136/ bmjopen-2017-015963).

Received 12 January 2017

Revised 8 June 2017

Accepted 10 July 2017
CrossMark

Department of Psychology, Stockholm University, Stockholm, Sweden

Correspondence to Jonas Rafi;

Jonas.rafi@sychology.su.se

\section{ABSTRACT}

Introduction Despite being considered a public health problem, no prevention programme for problem gambling in workplace settings has been scientifically evaluated. This study aims to fill a critical gap in the field of problem gambling by implementing and evaluating a large-scale prevention programme in organisations.

Methods and analysis Ten organisations, with a total of $n=549$ managers and $n=8572$ employees, will be randomised to either receiving a prevention programme or to a waitlist control condition. Measurements will be collected at the baseline and 3,12 and 24 months after intervention. The primary outcome of interest is the managers' inclination to act when worried or suspicious about an employee's problem gambling or other harmful use. Additional outcomes of interest include the Problem Gambling Severity Index and gambling habits in both managers and employees. Furthermore, qualitative analyses of the responses from semistructured interviews with managers will be performed.

Ethics and dissemination This study has been approved by the regional ethics board of Stockholm, Sweden, and it will contribute to the body of knowledge concerning prevention of problem gambling. The findings will be published in peer-reviewed, open-access journals. Trial registration number NCT02925286; Pre-results.

\section{INTRODUCTION}

Problem gambling (PG) is characterised by gambling-related behaviours that lead to negative consequences for the gambler, significant others or the community. ${ }^{1} \mathrm{PG}$ may lead to both social and economic losses ${ }^{1}$ and has been associated with poor general health ${ }^{2}$ and elevated rates of suicidal thoughts and acts. ${ }^{3}$ Furthermore, PG is associated with a higher prevalence of anxiety and depression compared with the normal population. ${ }^{5}$ In Sweden, the estimated point prevalence of PG is approximately $2 \%$, and the condition is classified as a public health problem. ${ }^{6}$

As of today, effective treatments aimed at PG exist. ${ }^{78}$ However, according to a systematic review by the Public Health Agency of

\section{Strengths and limitations of this study}

- This study will be the first large-scale study to evaluate a preventive intervention for problem gambling in a workplace setting.

- A majority of measurements will be administered via the Iterapi platform, a secure and encrypted website, which minimises risk of data loss and missing items.

- Primarily, the main outcome measure consists of self-reported perceptions of the managers' own abilities, which may be more biased and unreliable compared with behavioural measures.

- Survey response rates are generally low, making it difficult to draw conclusions.

- Since this study is conducted at organisations as opposed to a clinical setting, holding all environmental variables constant is more difficult.

Sweden, only $5 \%-12 \%$ of problem gamblers seek treatment, ${ }^{9}$ and research on treatments on PG show that not all gamblers benefit from treatment. $^{10} 11$ Furthermore, since the individuals and their significant others may have experienced severe consequences (eg, accumulated debts, divorce) before entering treatment, there is a need for effective preventive measures. ${ }^{12}$ According to the Swedish National Institute for Public Health, developing, evaluating and implementing methods that prevent PG is one the most important strategic national measures to minimise PG. ${ }^{13}$

Despite being important in public health matters, preventive interventions aimed at PG are scarce, ${ }^{9}$ and the fundamental aspects of effective preventive interventions are not yet defined. ${ }^{13}{ }^{14}$ Moreover, it is necessary to implement preventive interventions and to evaluate whether they lead to the desired effect. ${ }^{15}$ Some researchers have examined the effect of preventing problem gambling in schools and found that prevention programmes may be effective in reducing gambling. ${ }^{16}{ }^{17}$ However, to 
our knowledge, no evaluation of preventive interventions for PG has been conducted in workplace settings. Workplace interventions aimed at preventing other types of addiction have, however, shown promising results. For instance, Pidd $e t a l^{18}$ showed that the presence of alcohol and drug policies at Australian workplaces was associated with lower odds for the presence of alcohol and drug problems, even more so when it comes to policies focusing on the use of substances as well as on getting assistance following the identified use. Although the authors conclude that they cannot be sure whether the lower odds were caused by the policy interventions or some other variable, the findings are, in combination with the increased demand for evaluating preventive interventions, worth exploring. An interview study, conducted by Binde, ${ }^{19}$ has shown that professionals in the field of prevention and treatment of harmful use see clear workplace policies for both managers (for engaging in a conversation and following a specific action plan) and non-managerial employees (for reporting about one's suspicions of a colleague's harmful use) as a potentially useful tool to implement in a workplace setting. To meet the present societal needs on evaluated prevention programmes, the Public Health Agency of Sweden has funded the evaluation of such an initiative delivered by Alna, an organisation that provides addiction prevention services to workplaces. This research will be the first large-scale study of its kind to evaluate a preventive intervention for PG in a workplace setting.

In summary, there is a considerable knowledge gap regarding the effectiveness of $P G$ prevention in workplace settings. The evaluation of prevention programmes is important both in a public health perspective and for the organisations investing in preventive interventions. The current study aims to contribute with knowledge about the effects of such an intervention.

\section{Aims and hypotheses}

The aim of this study is to investigate the effects of a PG prevention programme in a workplace setting. It is hypothesised that the workplace PG prevention programme will result in (1) managers reporting a higher inclination to engage in a conversation with an employee when suspicion or worry about PG arises, (2) an increase in the employees' knowledge about where in the workplace one might get support regarding problem gambling, (3) an increased number of early interventions by the managers (eg, engage in a conversation) to help employees with PG or other types of harmful use, (4) managers and employees engaging in more sustainable gambling practices as measured by sum of Problem Gambling Severity Index (PGSI) scores, (5) fewer cases of PG among managers and employees as measured by PGSI categories and (6) managers reporting being more confident in handling gambling and PG in the workplace.

\section{METHODOLOGY}

The study will be a cluster randomised controlled trial with two arms: (1) the intervention programme and (2) a waitlist control that will be offered the same prevention programme after 1 year.

\section{Study population and recruitment}

The study population consists of employees at 10 organisations registered and active in Sweden, including $n=549$ managers and $n=8572$ non-managerial employees. The manager group includes supervisors, managers and human-resources (HR) staff working with personnel matters, rehabilitation and preventive measures. An overview of the included organisations and their employees is presented in table 1.

Alna carried out the recruitment of organisations in two ways: first, information was sent to all organisations

\begin{tabular}{llllll}
\hline \multicolumn{2}{l}{ Table 1} & Overview of each organisation's employees & & & \\
\hline ID & Context & Employee categories & $\mathbf{n}$ & \% of women & Mean age \\
\hline A & Authority & Administrators & 839 & 64 & 44.6 \\
B & Education & Teachers and researchers & 2035 & $*$ & ${ }^{*}$ \\
C & Education & Teachers and researchers & 849 & 55 & 46.6 \\
D & Dairy farming & Mixed office and manual workers & 403 & 22 & 42.3 \\
E & Staffing agency & Manual workers & 611 & 49 & 48 \\
F & Energy & Mixed office workers & 205 & 28 & 46 \\
G & Education & Teachers and researchers & 514 & 36 & $*$ \\
H & Municipality & Mixed office workers & 2631 & 74 & 45.9 \\
I & Education & Technicians, manual workers, & 404 & $*$ & $*$ \\
& Electronics manufacturer & Mixed office and manual workers & 81 & 19 & 36.2 \\
\hline
\end{tabular}

As the population of employees in a company often changes, the numbers presented here may not equal the actual population of employees included.

${ }^{*}$ Data have not been received from the organisation. 
in their customer register who had a minimum of 100 employees ( $\mathrm{n}=1967$ organisations); second, the organisations' representatives were informed of the study during a conference on gambling and PG in October 2015. The information consisted of a short overview and a rationale for the intervention. None of the organisations at the conference expressed interest; however, $n=12$ organisations from the customer register responded that they were interested; two of these organisations dropped out before randomisation.

On the individual participant level, all employees that the organisations sent contact information to were included in the study. Most organisations sent a complete list of employees in the organisations, whereas some organisations only chose specific sections. Before being able to answer the questionnaire, a consent form had to be signed before participating. See online supplementary file 1 for an English translation of the consent form. There were no exclusion criteria.

\section{Blinding}

As the waitlist condition is an assessment instead of a placebo condition, neither the organisations nor the researchers are blinded to the condition. However, since the questionnaire data are self-reported online without researcher involvement, no biases caused by the researchers' interpretations are expected. Questionnaire administration online has been shown to be reliable. ${ }^{20}$

\section{Intervention}

Alna created the intervention programme and its components in coordination with participating organisations from a previous project. ${ }^{21}$ The intervention is therefore based on their experience with working with organisations, rather than through theoretical frameworks. The intervention consists of two components: (1) implementation of a workplace gambling policy and (2) skill-development training.

The first component, policy implementation, consists of helping the organisation HR managers to strengthen or create and implement workplace policies for different types of addiction. The work on policies is based on Alna's manual 'Template for work with gambling and problem gambling at the workplace'. ${ }^{22}$ The manual, which is only available in Swedish, gives examples of what should be included in a policy based on Alna's earlier work on policies (eg, responsibilities, operative guidelines and rehabilitation). The policy work is performed together with HR and managers on three to four occasions (depending on organisation availability), each lasting 2 hours. The number of participants ranges between 7 and 50 .

The second component is a skill-development initiative in which Alna provides the managers with two 3.5-hour training sessions regarding addiction in general and gambling in particular. See box for the main topics covered in the training. Due to the varying availability of the organisations and their managers, the time between the two sessions ranges from 1 to 60 days. At the end of
Box Main topics covered during the two skilldevelopment workshops

- Different types of 'use': covers the difference between use, risk use, harmful use and addiction, particularly regarding gambling.

- Prevalence: statistics on the prevalence and change over time regarding gambling and problem gambling in Sweden.

- Risk factors: risk and protective factors in the workplace, regarding problem gambling and other harmful use.

- Conducting a conversation: the importance of using conversation as a tool. How to prepare, conduct and evaluate a conversation regarding potential harmful use.

- Signals: behaviours and signs that may signal problem gambling or other harmful use.

- Workplace cultures and policies: how workplace cultures may relate to harmful use. Benefits of having implemented workplace policies.

- Roles and responsibilities: discussion of roles and responsibilities for the organisation, managers and employees.

- Usage of the 'checklist': participants are shown the checklist and informed of its purpose.

- Dilemmas: covers different dilemmas by discussing ambiguous cases.

the second session, the "checklist for managers regarding problem gambling' is introduced, which is a tool Alna provides to help managers remember gambling topics when needed. ${ }^{23}$ The purpose of the checklist is to guide managers in how to act and how to interpret signs of behaviours that may negatively affect the employee and the workplace. The checklist consists of 22 questions and proposes actions, depending on how the questions were answered.

The group of Alna consultants conducting the prevention programme consists of $n=5$ consultants who have been working for Alna between 3 and 7 years.

\section{Waitlist condition}

Organisations in the waitlist condition will be on hold for 1 year while providing the same measurements as the intervention group. Thereafter, they will be offered to receive the intervention. See figure 1 for a study procedure flowchart.

\section{Outcome measures and data collection}

The primary outcome measure will be the managers' selfrated inclination to engage in a conversation (hypothesis 1) with employees who they worry or suspect may have a problem with gambling or other harmful use. This is represented as a question with a 1-to-10 scale in a self-report questionnaire developed specifically for this project (see online supplementary file 2 for an English translation of the questionnaire). Other items of the self-report questionnaire are discussed below.

The questionnaire consists of 20 items (including PGSI) and an additional 7 items in the managerial version. The items include measures regarding outcomes and demographics. Employees' and managers' knowledge about where to get support regarding PG (hypothesis 2) and other harmful use is investigated by using yes/ 
Months since

pre-measurement

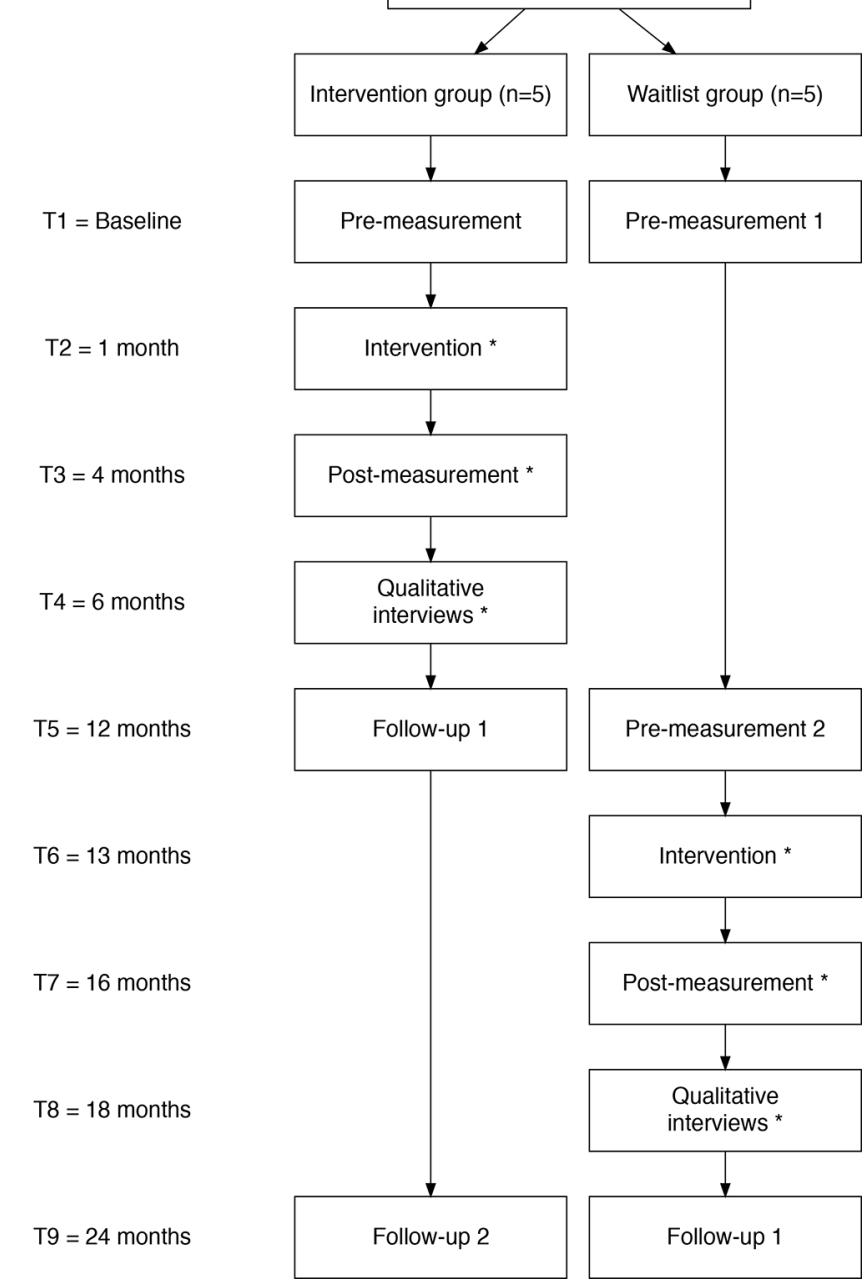

Figure 1 Study procedure flowchart and timeline. * indicates managerial event only.

no/unsure questions. Likewise, whether harmful use in the workplace has made them worried or made them take action (hypothesis 3) is also investigated using a yes/ no/unsure question. The PGSI will be used to explore changes in managers' and employees' gambling practices (hypothesis 4), using sum of PGSI score as outcome. Furthermore, the PGSI will be used to investigate levels of gambling problems among employees and managers (hypothesis 5), where the total score of 0 points indicates non-problem gambling; 1 to 2 points, low-risk gambling; 3 to 7 points, moderate-risk gambling; and 8 and above, problem gambling. PG is defined as a PGSI score of $\geq 8$. The PGSI has shown good psychometric properties $^{24} 25$ and is being used in the Swedish national longitudinal epidemiological study. ${ }^{26}$ Other items in the self-report questionnaire include (1) employees' and managers' knowledge of available policies regarding PG and other harmful use (yes/no/unsure) in their respective organisation (yes/no/unsure), (2) whether harmful use in the workplace has made them worried or made them take action (yes/no), (3) if they know about someone in the workplace who gambles or plays games during work (yes/no), (4) their own gambling habits (frequency of play offline/offline for the last 1 and 12 months) and (5) their age and gender. To enable comparison to other Swedish studies, the questions regarding gambling habits have been adopted from the Swedish Longitudinal Gambling Study (SWELOGS ${ }^{27}$ ).

In addition to the secondary outcomes aimed at both managers and employees, the self-report questionnaire sent to the managers includes some additional questions that are present only in the managers' questionnaires. These include the managers' self-perceived knowledge about gambling and problem gambling, and their perceived knowledge about how to act if a concern or suspicion about PG arises (hypothesis 6). Furthermore, semistructured interviews will be performed with a number of managers from the organisations in the project, thereby allowing a more in-depth analysis of their experiences of the intervention. The interviews will also aim to gather information about the nature of early interventions given (hypothesis 5 ).

The questionnaire is based on Alna's previous study, ${ }^{21}$ with the addition of the managerial questions and PGSI. After the questionnaire was completed, it was pilot tested by using a small group $(n=5)$ of individuals who were either psychologists or researchers.

\section{Data collection and protection}

A majority of the questionnaires will be administered online. The organisations will deliver the list of participant emails and demographics to the researchers. Thereafter, the online questionnaires will be sent via an email that includes a unique web link. Participants will be directed to a secure and encrypted website where they can answer the questionnaire. ${ }^{28}$ Therefore, the probability that the data are wrongly encoded or disappear can be considered low. Furthermore, the website requires that participants answer all questions before submitting. This might minimise the risk of missing items; however, this means that some participants may choose not to submit the questionnaire. Only the researchers involved will be able to access the database where the questionnaire information is stored. The data will be collected and stored in accordance with the Swedish Personal Data Act. To increase the number of responders, email reminders will be sent out a maximum of four times, with 5 to 7 days between each reminder.

Because not all participants among the employees have an organisation email address, several participants $(n=611)$ will instead have to use a shared workspace computer to access the questionnaire. However, they will still be directed to the secure website. To keep track of the participants using the workplace computer, the participants will be instructed to create a code based on their names and their day of birth. For example, John Doe, who was born on 6 June 1950, would enter JD06. This ensures that they will be able to fill in the same code during the follow-ups without having to remember or store anything. When completing the questionnaire, they will be assigned 
a randomised participant ID. This ID will be used instead of the participants' own code to ensure total anonymisation of the participants.

Furthermore, $n=827$ participants, divided over three organisations, have no effective way of setting up a computer for participants without email addresses. These participants will be handed printed questionnaires with a pre-assigned ID number and no personal information. Representatives from the organisations will distribute and collect the questionnaires. The questionnaires will then be handed to Alna, who then enters the data into a computer file and distributes the file to the researchers.

Lastly, in terms of the semistructured interviews, the responses will be transcribed in verbatim and analysed qualitatively. However, all personal details and sensitive information will be coded to ensure anonymity, and the original recording deleted following the transcription process.

A Standard Protocol Items: Recommendations for Interventional Trials $\left(\right.$ SPIRIT $^{29}$ ) checklist is provided in online supplementary file 3 .

\section{Randomisation}

In order to avoid contamination between the intervention and waitlist group, randomisation is done at the workplace level. Organisations will be randomised to one of the two groups. Randomisation will be conducted using the true random number service (random.org). Since two organisations are noticeably larger than the others, these will be randomised separate from the others to ensure that they are divided evenly. A researcher with no relation to the study will conduct the randomisation before the baseline measures are collected, ensuring minimal bias.

\section{Statistical analyses}

Because the study design inherits a hierarchical structure and, therefore, violates the assumptions of independent samples required for variance analysis, statistical analyses will be conducted within a linear mixed-model framework to model the variability on different levels. ${ }^{30}$ The authors will perform all the statistical analyses. Each outcome corresponds to a questionnaire question (see Outcome measures and data collection section), and the change in each corresponding question from baseline to post and follow-up will be analysed. Since Alna has a vested interest in the study results, it will not be involved in any part of the analyses or the subsequent discussion.

\section{Handling of attrition}

Statistical analyses will be performed using the intentionto-treat approach. ${ }^{31}$ According to a meta-analysis on the survey response rate in organisations, ${ }^{32}$ the mean response rate is as low as $35.7 \%(\mathrm{SD}=18.8 \%)$. Therefore, despite efforts to increase the response rate, it is expected to be low in the current project. The necessary steps to handle missing data with minimal bias depend on what assumptions can be made of the data that are missing. ${ }^{33}{ }^{34}$ Since the organisations will provide some demographic data on the participants, we expect to be able to perform a sensitivity analysis to explore potential differences between the complete sample and the sample of responders. Although recommended measures may vary depending on what assumptions can be made of the missing data, multiple imputation or full-information maximum-likelihood techniques are usually recommended. ${ }^{35} 36$

\section{Sample size}

As the data will be fitted using a repeated measures hierarchical linear model ${ }^{37}$ and there were neither a pilot study nor earlier relevant research to estimate the effect size, power was derived from a simulation study by Heo and Leon. ${ }^{38}$ Given the 10 clusters with an average of 36 managers and measurements on three occasions, an effect size of Cohen's $d=0.30$ should be detectable, given $80 \%$ power. This holds even if correlations on the managerial level are as high as 0.60 .

\section{Qualitative analysis}

Semistructured interviews will be conducted with a number of managers from the organisations in the project. The aim of the interviews is to collect information on the participating managers' experiences from the intervention that perhaps were not conveyed in the questionnaire. An interview guide will be created and used in order to ascertain that questions are raised regarding specific parts of the intervention programme, for example, implementation, yet still allowing the managers to respond freely and openly. The responses from the semistructured interviews will then be transcribed in verbatim and analysed using thematic analysis following the steps provided by Braun and Clarke. ${ }^{39}$ This will allow an in-depth exploration of the recurrent themes concerning particular concepts or phenomena, as perceived by the respondents themselves.

\section{DISCUSSION}

To our knowledge, this will be the first study to investigate the effectiveness of a large-scale prevention programme for PG in a workplace setting and thus break new ground in creating a basis for evidence-based policies for PG. If successful, the strategy of preventing PG in the normal population will be an important complement to the traditional approach of finding and treating high-risk individuals where great harm may already have occurred.

Conducting studies on PG prevention in a workplace setting may help framing it as a public health issue worth attention and treatment. This could potentially lead to more individuals seeking treatment. The strategy of aiming a prevention intervention towards the general population instead of high-risk populations is a key principle in modern public health approaches. ${ }^{40}$ However, the principle has not yet been explored in gambling interventions. ${ }^{41}$ Regardless of the intervention outcome, the results will be of high value to a variety of organisations, policy-makers, and the health and welfare authorities. 


\section{Limitations}

Several limitations exist. Primarily, the main outcome measure consists of self-reported perceptions of the manager's own abilities, which may be more biased and unreliable compared with behavioural measures. The broad nature of prevention and policy programme, combined with a large sample and low PG incidence, also makes it difficult to determine relevant evaluation measures. In addition, themes from the qualitative analysis may be hard to transfer to other organisational settings than those included in the project.

Some organisations will also likely have low response rates, making it difficult to interpret the validity of the results and to compare effects between organisations. Furthermore, the organisation employees may differ considerably in attitude and acceptance towards participating, which may affect response rates and other factors that are beyond the researchers' control. Rules, regulations and workplace culture may affect the intervention and its outcomes. Despite adding ecological validity, these factors may lead to differences between the organisations that are not known or considered.

Furthermore, as the intervention is delivered at workplace settings, possible effects will not be generalisable to the non-working population.

\section{Ethics and dissemination}

The Regional Ethics Board in Stockholm, Sweden (Dnr: 2016/1208-31/5), approved the study protocol. All participants will be informed that participating is voluntary. The informed consent is presented to the participants at the survey website before the survey is shown. Therefore, only participants who agree to the informed consent will be able to proceed to the study questionnaires.

Participants' anonymity will be ensured by using randomised ID tags, by restricting questionnaire responses for age intervals instead of exact age and by restricting data access to only the research group. Data will be clustered by groups; thus, the possibility of inferring participant identities will be minimised.

Results will be published in open-access journals and presented at conferences. Furthermore, the Public Health Agency of Sweden will write reports on the results. Our aim is to share as much data as possible while still respecting the participants' integrity.

Acknowledgements We thank Alna for providing requested information on programme details, and Kristoffer Magnusson for guidance on power estimates.

Contributors JR drafted the manuscript. JR, El, AR and PC all participated in the study design and coordination. All authors read, revised and approved the final manuscript.

Funding The Public Health Agency of Sweden have given Alna funding to conduct this project. Alna, in turn, has used parts of the funding to entrust the authors to conduct an independent evaluation of the project.

Competing interests None declared.

Ethics approval Regional Ethics Board in Stockholm.

Provenance and peer review Not commissioned; externally peer reviewed.
Data sharing statement To create as much value as possible from our data, we aim to publish the gathered data on an open-access repository. However, since the data may be sensitive, only averages will be available.

Open Access This is an Open Access article distributed in accordance with the Creative Commons Attribution Non Commercial (CC BY-NC 4.0) license, which permits others to distribute, remix, adapt, build upon this work non-commercially, and license their derivative works on different terms, provided the original work is properly cited and the use is non-commercial. See: http://creativecommons.org/ licenses/by-nc/4.0/

(C) Article author(s) (or their employer(s) unless otherwise stated in the text of the article) 2017. All rights reserved. No commercial use is permitted unless otherwise expressly granted.

\section{REFERENCES}

1. Neal P, Delfabbro P, O'Neil M. Problem gambling and harm: towards a national definition. 2005.

2. Morasco BJ, Vom Eigen KA, Petry NM. Severity of gambling is associated with physical and emotional health in urban primary care patients. Gen Hosp Psychiatry 2006;28(2):94-100.

3. Grant JE, Derbyshire K, Leppink E, et al. Suicidality in non-treatment seeking young adults with subsyndromal gambling disorder. Psychiatr Q 2014;85:513-22.

4. Stein GN, Pretorius A, Stein DJ, et al. The association between pathological gambling and suicidality in treatment-seeking pathological gamblers in South Africa. Ann Clin Psychiatry 2016;28:43-50.

5. Lorains FK, Cowlishaw S, Thomas SA. Prevalence of comorbid disorders in problem and pathological gambling: systematic review and meta-analysis of population surveys. Addiction 2011;106:490-8.

6. Abbott MW, Romild U, Volberg RA. Gambling and problem gambling in Sweden: changes between 1998 and 2009. J Gambl Stud 2014;30:985-99.

7. Cowlishaw S, Merkouris S, Dowling N, et al. Psychological therapies for pathological and problem gambling. Cochrane Database Syst Rev 2012;11:CD008937.

8. Yakovenko I, Quigley L, Hemmelgarn BR, et al. The efficacy of motivational interviewing for disordered gambling: systematic review and meta-analysis. Addict Behav 2015;43:72-82.

9. Folkhälsoinstitut S. Att förebygga spelproblem [Preventing problem gambling]. 2010.

10. Yakovenko I, Hodgins DC. Latest developments in treatment for disordered gambling: review and critical evaluation of outcome studies. Curr Addict Rep 2016;3:299-306.

11. Andersson G, Cuijpers P, Carlbring P, et al. Guided internet-based vs. face-to-face cognitive behavior therapy for psychiatric and somatic disorders: a systematic review and meta-analysis. World Psychiatry 2014;13:288-95

12. Svensson J, Romild U, Shepherdson E. The concerned significant others of people with gambling problems in a national representative sample in Sweden-a 1 year follow-up study. BMC Public Health 2013;13:1087.

13. Statens folkhälsoinstitut. Spel: Kunskapsunderlag För Folkhälsopolitisk Rapport 2010. Östersund: Statens folkhälsoinstitut; 2011, Rapport nr: R 2011:19.

14. Ariyabuddhiphongs V. Problem gambling prevention: before, during, and after measures. Int J Ment Health Addict 2013;11:568-82.

15. Rodgers B, Suomi A, Davidson T. Preventive interventions for gambling: a public health perspective. 2015.

16. Donati MA, Primi C, Chiesi F. Prevention of problematic gambling behavior among adolescents: testing the efficacy of an integrative intervention. J Gambl Stud 2014;30:803-18.

17. Williams RJ, Wood RT, Currie SR. Stacked Deck: an effective, school-based program for the prevention of problem gambling. $J$ Prim Prev 2010;31:109-25.

18. Pidd K, Kostadinov V, Roche A. Do workplace policies work? An examination of the relationship between alcohol and other drug policies and workers' substance use. Int J Drug Policy 2016;28:48-54.

19. Binde P. Preventing and responding to gambling-related harm and crime in the workplace. Nordic Studies on Alcohol and Drugs 2016;33:247-65.

20. Carlbring P, Brunt S, Bohman S, et al. Internet vs. paper and pencil administration of questionnaires commonly used in panic/ agoraphobia research. Comput Human Behav 2007;23:1421-34. 
21. Alna. Spelförebyggande insatser för arbetslivet: fokus på unga vuxna [Gambling prevention in the workplace, targeting young adults]. Stockholm: Alna \& Rodeo, 2015.

22. Alna. Mall för arbete med spel och spelproblem på arbetsplatsen [Template for working with gambling and problem gambling in the workplace]. 2015.

23. Nyqvist M. Checklista för chefer vid spelproblem [Problem gambling checklist for managers]. 2015.

24. Wynne H, Ferris J. The Canadian problem gambling index: final report. 2001.

25. Holtgraves T. Evaluating the problem gambling severity index. $J$ Gambl Stud 2009;25:105-20.

26. Romild U, Volberg R, Abbott M. The Swedish Longitudinal Gambling Study (Swelogs): design and methods of the epidemiological (EP-) track. Int J Methods Psychiatr Res 2014;23:372-86.

27. Statens Folkhälsoinstitut. Spel om pengar och spelproblem i Sverige 2009/2010 [Gambling and problem gambling in Sweden 2009/2010]. 2012.

28. Vlaescu G, Alasjö A, Miloff A, et al. Features and functionality of the Iterapi platform for internet-based psychological treatment. Internet Interv 2016;6:107-14.

29. Chan AW, Tetzlaff JM, Gøtzsche PC, et al. SPIRIT 2013 explanation and elaboration: guidance for protocols of clinical trials. BMJ 2013;346:e7586.

30. Snijders TAB. Multilevel analysis. In: Lovric M, ed. International encyclopedia of statistical science. Berlin, Heidelberg: Springer, 2011:879-82
31. Hollis S, Campbell F. What is meant by intention to treat analysis? Survey of published randomised controlled trials. BMJ 1999;319:670-4.

32. Baruch $\mathrm{Y}$, Holtom BC. Survey response rate levels and trends in organizational research. Human Relations 2008;61:1139-60.

33. Brick JM, Kalton G. Handling missing data in survey research. Stat Methods Med Res 1996;5:215-38.

34. Graham JW. Missing data-analysis and design. 2007.

35. Sterne JA, White IR, Carlin JB, et al. Multiple imputation for missing data in epidemiological and clinical research: potential and pitfalls. BMJ 2009;338:b2393.

36. Eekhout I, Enders CK, Twisk JW, et al. Including auxiliary item information in longitudinal data analyses improved handling missing questionnaire outcome data. J Clin Epidemiol 2015;68:637-45

37. Hesser $\mathrm{H}$. Modeling individual differences in randomized experiments using growth models: recommendations for design, statistical analysis and reporting of results of internet interventions. Internet Interv 2015;2:110-20.

38. Heo M, Leon AC. Sample size requirements to detect an intervention by time interaction in longitudinal cluster randomized clinical trials Moonseong. 2009;28:1017-27.

39. Braun V, Clarke V. Using thematic analysis in psychology. Qual Res Psychol 2006:3:77-101.

40. Rose G. The strategy of preventive medicine. University Press: Oxford, 1992.

41. Canale N, Vieno A, Griffiths MD. the extent and distribution of gambling-related harms and the prevention paradox in a British population survey. J Behav Addict 2016;5:204-12. 